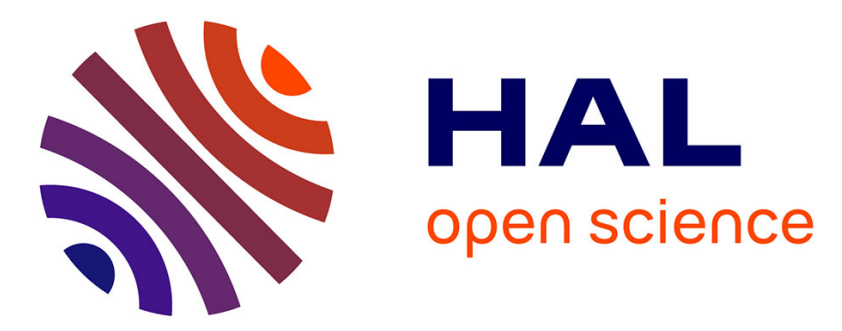

\title{
Marine signature taxa and core microbial community stability along latitudinal and vertical gradients in sediments of the deepest freshwater lake
}

Guillaume Reboul, David Moreira, Nataliia V Annenkova, Paola Bertolino, Konstantin E Vershinin, Purificación López-García

\section{To cite this version:}

Guillaume Reboul, David Moreira, Nataliia V Annenkova, Paola Bertolino, Konstantin E Vershinin, et al.. Marine signature taxa and core microbial community stability along latitudinal and vertical gradients in sediments of the deepest freshwater lake. ISME Journal, 2021. hal-03384282

\section{HAL Id: hal-03384282 \\ https://hal.science/hal-03384282}

Submitted on 18 Oct 2021

HAL is a multi-disciplinary open access archive for the deposit and dissemination of scientific research documents, whether they are published or not. The documents may come from teaching and research institutions in France or abroad, or from public or private research centers.
L'archive ouverte pluridisciplinaire HAL, est destinée au dépôt et à la diffusion de documents scientifiques de niveau recherche, publiés ou non, émanant des établissements d'enseignement et de recherche français ou étrangers, des laboratoires publics ou privés. 
Marine signature taxa and core microbial community stability along latitudinal and vertical gradients in sediments of the deepest freshwater lake

5 Guillaume Reboul ${ }^{1}$, David Moreira ${ }^{1}$, Nataliia V. Annenkova ${ }^{2}$, Paola Bertolino ${ }^{1}$, Konstantin E. Vershinin ${ }^{2}$ and Purificación López-García ${ }^{1}$

${ }^{1}$ Ecologie Systématique Evolution, Centre National de la Recherche Scientifique - CNRS, Université Paris-Saclay, AgroParisTech, Orsay, France

$10{ }^{2}$ Limnological Institute, Siberian Branch of the Russian Academy of Sciences, Irkutsk, Russia

For correspondence: puri.lopez@u-psud.fr

15

Running title: Benthic microbial communities of Lake Baikal 
Lake Baikal is the deepest ( $1.6 \mathrm{~km}$ ) and most voluminous freshwater reservoir on Earth. Compared to plankton, its benthos remains poorly explored. Here, we ask whether latitude and/or depth determine benthic microbial community structure and how Baikal communities compare to those of other freshwater, brackish and marine sediments. To answer, we collected sediment upper layers $(0-1 \mathrm{~cm})$ across a $\sim 600 \mathrm{~km}$ North-South transect covering the three basins of the lake and from littoral to bathybenthic depths ( 0.5 to $1450 \mathrm{~m}$ ). Metabarcoding of $16 \mathrm{~S}$ and $18 \mathrm{~S}$ rRNA genes revealed communities with high richness and evenness where rare operational taxonomic units (OTUs) collectively dominated. Archaea represented up to $25 \%$ or prokaryotic sequences. Baikal sediments harbored typically marine eukaryotic and prokaryotic OTUs recently identified in some lakes (diplonemids, Bolidophyceae, Mamiellales, SAR202, marine-like Synechococcus, Pelagibacterales) but also SAR324, Syndiniales and Radiolaria. We hypothesize that, beyond the salinity barrier, adaptation to oligotrophy explains the presence of these otherwise typically marine lineages. Baikal core benthic communities were relatively stable across sites and seemed not determined by depth or latitude. Comparative analyses with other freshwater, brackish and marine prokaryotic sediment communities confirmed the distinctness of Baikal benthos, which include elements of similarity to marine and hydrothermally-influenced systems.

Keywords: Lake Baikal; benthos; 16S/18S rRNA metabarcoding; archaea; bacteria; protist; marine- 
Lake Baikal in Southern Siberia is the oldest ( $\sim 30$ Myr), deepest ( $1650 \mathrm{~m}$ maximum and $750 \mathrm{~m}$ and average depth), and largest (by volume, $\sim 23000 \mathrm{~km}^{3}$ ) freshwater lake on Earth. This unique ecosystem is akin, in several respects, to sea environments [1]. Its surface freezes in winter, which triggers coastal downwelling and deep-water ventilation. Consequently, its water body remains cold $\left(\sim 4^{\circ} \mathrm{C}\right)$, oxygenrich and ultra-oligotrophic [2]. Deep, cold and associated with a rifting zone, Lake Baikal host methane hydrates [3]. Geographically, the Academician Ridge and the Selenga river delta delimitate three basins: Northern, Central, and Southern [1]. Lake Baikal hosts many endemic metazoan species [2]. Molecular analyses of prokaryotic (e.g.[4-6]) and microbial eukaryotic (e.g.[7-9]) diversity have been mostly carried out in plankton. Comparatively, benthic microbial communities remain poorly known, with few studies of archaea and bacteria in bottom, often seepage-influenced, sediments [10] and of sedimentassociated eukaryotes [7]. Here, we asked how benthic communities vary spatially along latitudinal and vertical gradients in Lake Baikal and how they compare to those of other freshwater, brackish and marine ecosystems. To answer, we carried out a comparative study of Baikal benthic prokaryotic and eukaryotic microbial communities across a $\sim 600 \mathrm{~km}$ latitudinal North-South gradient traversing the three lake basins and from surface (littoral sediment) to the greatest depths (>1400 m) using a metabarcoding approach (Fig.1A; Supplementary Text). We purified DNA from the upper sediment layer (ca. 0-1 cm) and massively sequenced amplicons of 16S (V4-V5 region) and 18S (V4 region) rRNA genes. High-quality reads clustered in 33943 prokaryotic and 3351 eukaryotic SWARM-derived operational taxonomic units (OTUs), which did not reach saturation in accumulation curves (Supplementary Fig.1; Supplementary Text). Computed from rarefied sequence matrices, eukaryotic and, especially, prokaryotic communities exhibited high richness, diversity and evenness scores (Supplementary Tables 1-2). Therefore, Baikal sediments seem to harbor complex communities with no clear dominant species.

Archaea reached up to $25 \%$ relative abundance and, on average, $20 \%$ of the OTUs (Supplementary Fig.2). They encompassed diverse phyla, consistent with recent studies of seepage areas [10], with DPANN (Woesearchaeota and Pacearchaeota, now included in Nanoarchaeota) and TACK (Nitrososphaeria-Thaumarchaeota and, in some samples Bathyarchaeota) being the most abundant, followed by Euryarchaeota (Thermoprofundales/Marine Benthic Group-D and locally Methanofastidiosales/WS2A) (Fig.1A; Supplementary Figs.3-4; Supplementary Table 3). Free-living Thermoprofundales and Thaumarchaeota might be potential hosts for the parasitic/symbiotic DPANN [11]. Bacteria comprised a wide variety of phyla likely involved in complex N, S and C cycling. PVC members (Verrucomicrobia and Planctomycetes) and Acidobacteriota were the most relatively abundant, followed by FCB (notably Bacteroidota, Latescibacteria, Gemmatimonadota), Proteobacteria, Chloroflexi and Nitrospinota (Fig.1A; Supplementary Fig.5). Proteobacteria (ca. 15\%) 
were little abundant compared to lake or marine surficial sediments, but akin subseafloor sediments [12]. Some biomass-degrading lineages, e.g. Phycisphaerae [13], Ignavibacteria [14] or Latescibacteria [15], suggest the contribution of thermophilic or seepage-associated microbes to Baikal benthic communities, possibly along faulting zones. Benthic protists were dominated by Stramenopiles and Alveolata (Fig.1A). Alveolates comprised mostly ciliates, dinoflagellates and Syndiniales (Supplementary Table 4). Stramenopiles included heterotrophic lineages (labyrinthulids, amphifilidae/thraustochytridlike, oomycetes, MAST) and ochrophyte algae (chrysophytes, diatoms), which sediment from the water column [16]. Compared to prokaryotes, benthic protists were rare in seeping zones (BK22S), as obtaining amplicons was difficult. The abundance (percentage of reads) and diversity (percentage of OTUs) patterns of rare versus abundant OTUs across phyla were rather similar for eukaryotes and rare prokaryotic OTUs, whereas the diversity of abundant prokaryotic OTUs was remarkably constant across sites (Supplementary Figs.6-7), suggesting a relatively stable core of dominant OTUs.

To see whether the depth and/or the latitude determined benthic microbial community structure, we performed an NMDS analysis based on dissimilarity matrices of OTU frequencies. However, we observed no obvious pattern discriminating samples according to basin or depth (Fig.1B). Although two samples of intermediate depth $(100-800 \mathrm{~m})$ appeared to segregate on along axis 1 , surface $(<100 \mathrm{~m})$ and deep (>800 m) samples appeared mixed. This pattern was almost superimposable to that observed for prokaryotic OTUs, whereas eukaryotic OTUs seemed to segregate better surface from deep samples (Supplementary Fig.8). PERMANOVA analyses confirmed no significant discrimination of prokaryotic and eukaryotic communities at the OTU level by latitude and only marginal significance for depth (prokaryotes, $R^{2}=0.23, p=0.01$; eukaryotes, $R^{2}=0.19, p=0.07$ ) (Supplementary Table 5). Since this marginal effect of depth might be due to the large collective dominance of rare OTUs ( $\sim 0 \%$ prokaryotic and $~ 85 \%$ eukaryotic OTUs; Fig.1C, upper-right inset), we also carried out NMDS and PERMANOVA analyses on i) dissimilarity matrices at high-rank taxon, rather than OTU, level, ii) Jaccard distance matrix, iii) Unifrac distance matrix and iv) Null model Raup-Crick index. Again, prokaryotic and eukaryotic communities did not segregate by depth (Supplementary Figs.9-12). Phyla and depth categories did not significantly correlate for eukaryotes and only marginally for prokaryotes (Supplementary Tables 5-6). This situation contrasts with that of stratified plankton communities [8] and might be due to similar sediment environmental conditions, notably temperature, across lake depths. We detected a core of 44 prokaryotic OTUs shared by all the sediment samples that represented between $\sim 15 \%$ and $\sim 40 \%$ of prokaryotic abundance (Fig.1C). Their phylogenetic affiliation

110 fitted the overall prokaryotic phyla distribution, suggesting that, accompanying widely diverse rare OTUs, there is a stable core of benthic prokaryotic communities across basins and depths in Lake Baikal. 
At odds with the idea that marine-freshwater transitions rare [17], we identified 332 OTUs belonging to typical marine taxa across the lake (Fig.2A; Supplementary Table 7). Several of them belonged to groups already observed in freshwater systems, such as MAST clades, relatively abundant in Baikal, but also rarer diplonemids, Radiolaria (for which we identified several OTUs; Supplementary Fig.13), Mamiellales, Bolidophyceae and, among bacteria, marine-like Synechococcus, SAR202 Chloroflexi and Pelagibacterales. Some of them were recently observed in Lake Baikal $[8,9,18]$. Others have never been reported in freshwater ecosystems, such as diverse Syndiniales and SAR324 Deltaproteobacteria, metabolically flexible sulphur metabolizers often associated with submarine hydrothermal plumes [19].

120 To eliminate potential cross-contamination with marine samples during collection, handling or sequencing, we mined for other typical abundant marine taxa in our datasets. We detected neither thraustochytrids nor Prochlorococcus and Alteromonas (Fig.2A), reinforcing the conclusion that Baikal 'marine' OTUs are indeed autochthonous. Our study confirms and extends the presence of several typically marine prokaryotes and eukaryotes at low abundances in Lake Baikal, reinforcing the idea that

125 transition frequency between marine and freshwater habitats is underestimated [20]. This opens questions about the specific molecular adaptations to the very low salinity of Lake Baikal salinity $(0.0$ PSU) and suggests that oligotrophy and deep waters might be more important drivers than salinity for these lineages.

How do benthic Baikal communities compare to those of other aquatic ecosystems? Does the presence of Baikal marine taxa indicate intermediate ecological features between freshwater and marine environments? To answer, we retrieved 16S rRNA gene metabarcoding data from other freshwater, brackish and marine sediment samples (Supplementary Text; Supplementary Tables 8-9). An NMDS plot based on the high-rank taxa dissimilarity matrix showed that Lake Baikal sediment samples clustered away from other samples, albeit closer to freshwater systems (Fig.2B). PERMANOVA analyses revealed a significant effect of the size and salinity category $\left(R^{2}=0.45 ; p\right.$-value $\left.=10^{-4}\right)$ (Supplementary Table 5), partly driven by Acidobacteriota, PVC, Nitrospirota and Chloroflexi (Fig.2B-C). Therefore, the comparison of communities associated to upper-layer sediment in Lake Baikal and other benthic ecosystems across different depth and salinity ranges set it apart from other freshwater and marine systems. during our 2017 limnological cruise, and Philippe Deschamps for technical bioinformatic support. We thank the crew of the R/V G. Titov for their professionalism and efficiency onboard and the director of the Limnological Institute at Irkusk for logistical assistance. This research was funded by the European 
145 Research Council Grants ProtistWorld (322669, PL-G) and PlastEvol (787904, DM) as well as the Russian State grant 0345-2016-0009 (NVA).

Author contributions PLG, DM and NVA designed the work and organized the limnological cruise. PLG, GR, NVA and KEV collected sediment samples. PB and GR purified DNA and carried out PCR reactions for metabarcoding. GR carried out the bioinformatic analysis of amplicon sequences, statistical analyses and wrote an early draft of the manuscript. PLG wrote the final manuscript. All authors read, critically commented and approved the final manuscript.

\section{Compliance with ethical standards}

155 Conflict of interest The authors declare that they have no conflicts of interest.

\section{References}

1. UNDP-GEF. The ecological atlas of the Baikal basin. United Nations Office for Project Sercives (UNOPS): http://baikal.iwlearn.org/en. 2015. p 145.

160 2. Moore MV, Hampton SE, Izmest'eva LR, Silow EA, Peshkova EV, Pavlov BK. Climate change and the world's "Sacred Sea" - Lake Baikal, Siberia. Bioscience. 2009; 59: 405-417.

3. Granin NG, Aslamov IA, Kozlov VV, Makarov MM, Kirillin G, McGinnis DF et al. Methane hydrate emergence from Lake Baikal: direct observations, modelling, and hydrate footprints in seasonal ice cover. Sci Rep. 2019; 9: 19361.

165 4. Glöckner FO, Zaichikov E, Belkova N, Denissova L, Pernthaler J, Pernthaler A et al. Comparative 16S rRNA analysis of lake bacterioplankton reveals globally distributed phylogenetic clusters including an abundant group of actinobacteria. Appl Environ Microbiol. 2000; 66: 5053-5065.

5. Kurilkina MI, Zakharova YR, Galachyants YP, Petrova DP, Bukin YS, Domysheva VM et al. Bacterial community composition in the water column of the deepest freshwater Lake Baikal as determined by next-generation sequencing. FEMS Microbiol Ecol. 2016; 92.

6. Zakharenko AS, Galachyants YP, Morozov IV, Shubenkova OV, Morozov AA, Ivanov VG et al. Bacterial communities in areas of oil and methane seeps in pelagic of Lake Baikal. Microb Ecol. 2019; 78: 269285.

7. Yi Z, Berney C, Hartikainen H, Mahamdallie S, Gardner M, Boenigk J et al. High-throughput sequencing of microbial eukaryotes in Lake Baikal reveals ecologically differentiated communities and novel evolutionary radiations. FEMS Microbiol Ecol. 2017; 93: 10.

8. David GM, Moreira D, Reboul G, Annenkova NV, Galindo LJ, Bertolino P et al. Environmental drivers of plankton protist communities along latitudinal and vertical gradients in the oldest and deepest freshwater lake. Environ Microbiol. 2021; 23: 1436-1451.

180 9. Annenkova NV, Giner CR, Logares R. Tracing the origin of planktonic protists in an ancient lake. Microorganisms. 2020; 8. 
10. Lomakina AV, Mamaeva EV, Galachyants YP, Petrova DP, Pogodaeva TV, Shubenkova OV et al. Diversity of archaea in bottom sediments of the discharge areas with oil- and gas-bearing fluids in Lake Baikal. Geomicrobiol J. 2018; 35: 50-63.

11. Castelle CJ, Brown CT, Anantharaman K, Probst AJ, Huang RH, Banfield JF. Biosynthetic capacity, metabolic variety and unusual biology in the CPR and DPANN radiations. Nat Rev Microbiol. 2018; 16: 629-645.

12. Biddle JF, Fitz-Gibbon S, Schuster SC, Brenchley JE, House CH. Metagenomic signatures of the Peru Margin subseafloor biosphere show a genetically distinct environment. Proc Natl Acad Sci U S A. 2008; 105: 10583-11058.

13. Spring S, Bunk B, Sproer C, Rohde M, Klenk HP. Genome biology of a novel lineage of planctomycetes widespread in anoxic aquatic environments. Environ Microbiol. 2018; 20: 2438-2455.

14. Podosokorskaya OA, Kadnikov VV, Gavrilov SN, Mardanov AV, Merkel AY, Karnachuk OV et al. Characterization of Melioribacter roseus gen. nov., sp. nov., a novel facultatively anaerobic thermophilic cellulolytic bacterium from the class Ignavibacteria, and a proposal of a novel bacterial phylum Ignavibacteriae. Environ Microbiol. 2013; 15: 1759-1771.

15. Dombrowski N, Seitz KW, Teske AP, Baker BJ. Genomic insights into potential interdependencies in microbial hydrocarbon and nutrient cycling in hydrothermal sediments. Microbiome. 2017; 5: 106.

16. Roberts SL, Swann GEA, McGowan S, Panizzo VN, Vologina EG, Sturm M et al. Diatom evidence of 20th century ecosystem change in Lake Baikal, Siberia. PLoS One. 2018; 13: e0208765.

17. Mukherjee I, Hodoki Y, Okazaki Y, Fujinaga S, Ohbayashi K, Nakano SI. Widespread dominance of kinetoplastids and unexpected presence of diplonemids in deep freshwater lakes. Front Microbiol. 2019; 10: 2375.

18. Zemskaya TI, Cabello-Yeves PJ, Pavlova ON, Rodriguez-Valera F. Microorganisms of Lake Baikal-the deepest and most ancient lake on Earth. Appl Microbiol Biotechnol. 2020.

19. Sheik CS, Jain S, Dick GJ. Metabolic flexibility of enigmatic SAR324 revealed through metagenomics and metatranscriptomics. Environ Microbiol. 2014; 16: 304-317.

20. Paver SF, Muratore D, Newton RJ, Coleman ML. Reevaluating the salty divide: phylogenetic specificity of transitions between marine and freshwater systems. mSystems. 2018; 3. 
Figure Legends

Fig.1. Sampling points and overall prokaryotic and eukaryotic diversity in Baikal sediments. A, Bathymetric map of Lake Baikal showing the sampling sites and depths along the three major basins of the lake extending along the North-South latitude axis (left panel). Relative abundance of clean merged reads (CMRs) representing the major prokaryotic and eukaryotic taxa for each sampling location. The asterisk shows the average diversity derived from two replicates from the same sampling site, after nested PCR amplification. B, Non-metric multidimensional scaling (NMDS) of Bray-Curtis dissimilarities based on OTU frequencies of both prokaryotic and eukaryotic OTUs. Each point represents a different sample. Ellipses enclose all points per depth category: shallow (<100 m), medium (100-800 m), deep (>800 m). Samples from the different Baikal basins are indicated with different marker shapes. NMDS for only prokaryotic and eukaryotic communities are presented in Supplementary Figure 8. C, UpSetR plot (central panel) showing the number, phylogenetic affiliation (taxa indicated in bold in A) and relative abundance of OTUs within the core prokaryotic community shared by all Baikal sediment samples (left bar) or all the sediment samples but one (light grey dot; bars on the right). The bottomleft histogram shows the relative proportion (CMRs) of the prokaryotic core community in the total prokaryotic community of each sediment sample. The upper right inset shows the total number of shared prokaryotic and eukaryotic OTUs per groups of sediment samples.

Fig.2. Marine signature taxa and comparison of Lake Baikal with other freshwater, brackish and marine sediment communities. A, Presence (light blue) / absence (white) matrix of typical marine taxa identified in Lake Baikal sediments. Each row represents a sampling location and each column a taxon. The barcharts represent the sum of the detected CMRs (dark red) and OTUs (light red) per typical marine taxon (top) and sampling location (right). B, NMDS of sediment samples based on Bray-Curtis dissimilarities of bacterial high-rank taxa. Colored ellipses and symbols correspond to Baikal (light blue squares), other freshwater sediments (light green squares), brackish (red dots) and marine (dark blue triangles) sediment samples. C, Diversity barchart displaying the relative abundance of bacterial sequences in the different sediment samples (left) and the dendrogram (right) resulting from the corresponding clustering analysis based on the Bray-Curtis dissimilarity. Dendrogram leaves represent the NMDS points depicted in (B). 

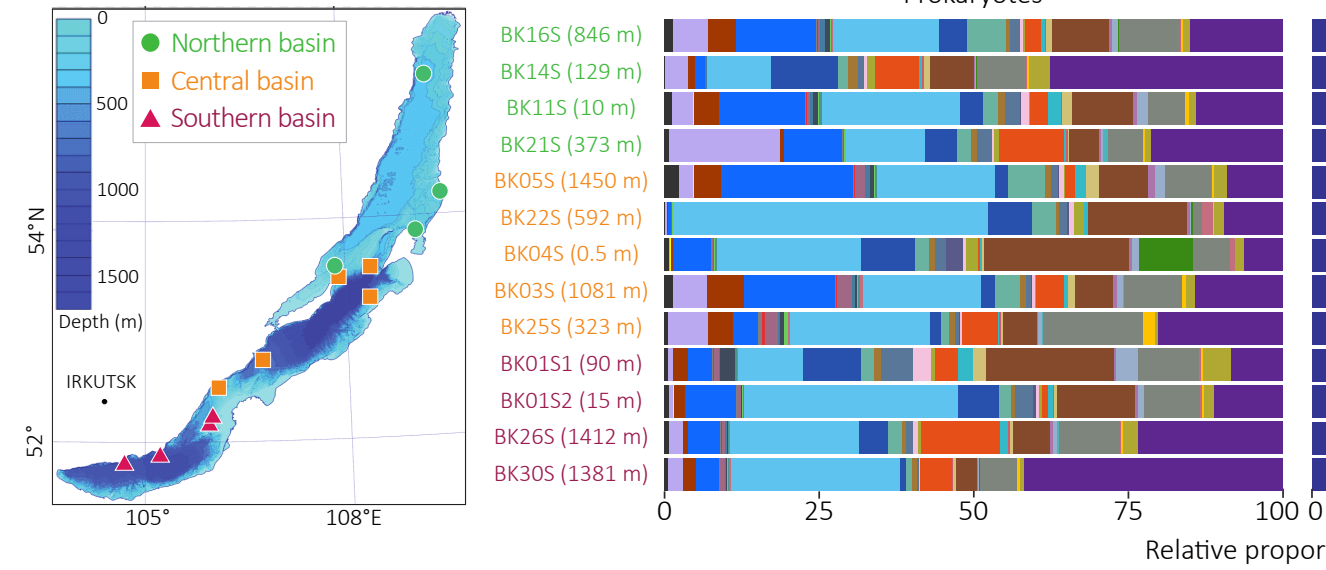

Eukaryotes

\begin{tabular}{llll} 
Archaea & Bacteria & \\
\cline { 2 - 3 } Unclassified Archaea & WS2 & Patescibacteria \\
\cline { 2 - 3 } TACK & WS1 & Other Bacteria \\
Other Archaea & WOR-1 & Nitrospirota \\
Euryarchaeota & Unclassified Bacteria & Nitrospinota \\
DPANN & Sva0485 & NB1-j \\
& Sumerlaeota & Myxococcota \\
& Spirochaetota & Methylomirabilota \\
& Schekmanbacteria & MBNT15 \\
& PVC & Hydrogenedentes \\
& Proteobacteria & Firmicutes
\end{tabular}

FCB
Elusimicrobiota
Desulfobacterota
Dadabacteria
Cyanobacteria
Chloroflexi
Bdellovibrionota
Armatimonadota
Actinobacteriota
Acidobacteriota

Eukaryotes

B

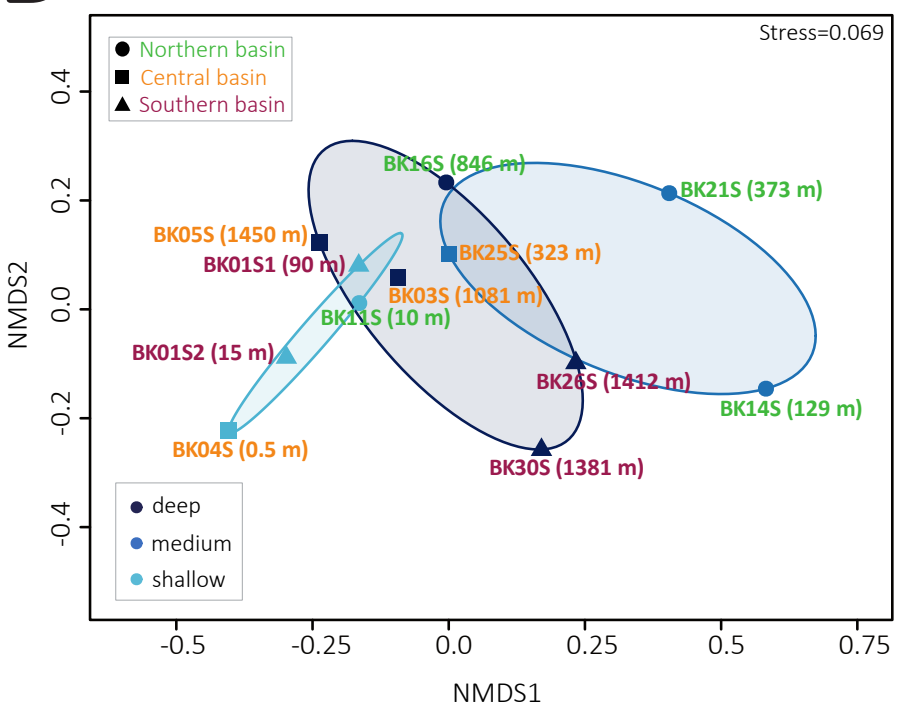

Relative abundance of shared OTUs

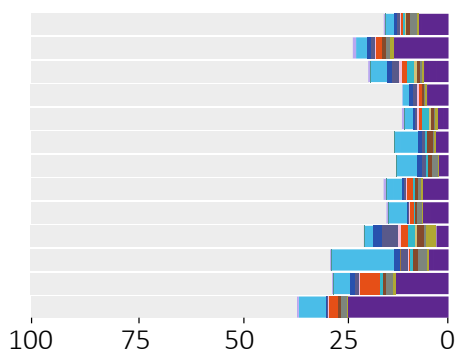

BK16S (846 m) BK14S (129 m) BK11S (10 m) BK21S (373 m) BK05S $(1450 \mathrm{~m})$ BK22S $(592 \mathrm{~m})$ BKO3S (1081 m) BK25S (323 m) BK01S1 $(90 \mathrm{~m})$ BK01S2 $(15 \mathrm{~m})$ BK26S (1412 m) BK30S (1381 m)

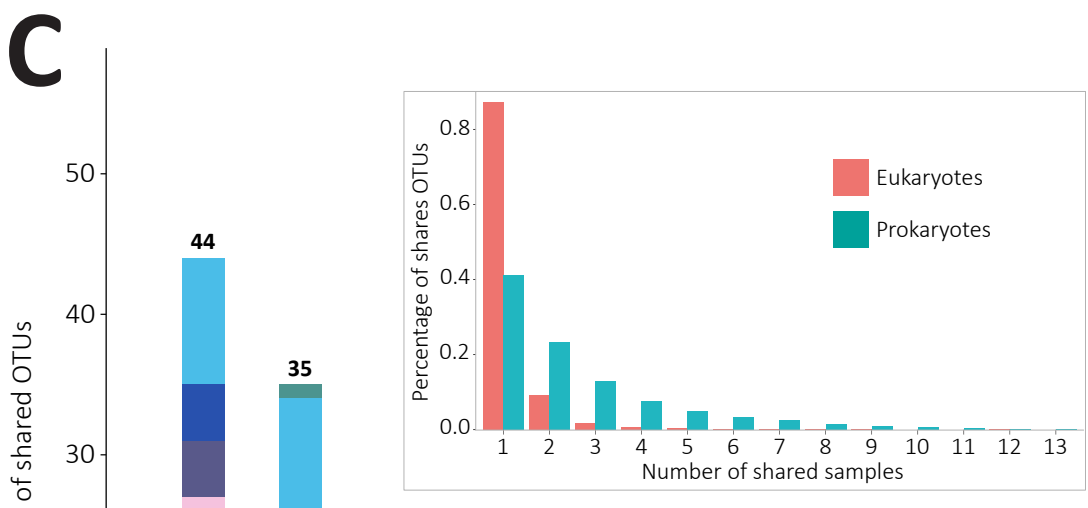

Figure 1. Reboul et al.

$\begin{array}{ll}\text { Stramenopiles } & \text { Archaeplastida } \\ \text { Rhizaria } & \text { Apusomonads } \\ \text { Opisthokonta } & \text { Amoebozoa } \\ \text { Hacrobia } & \text { Alveolata }\end{array}$

Excavata
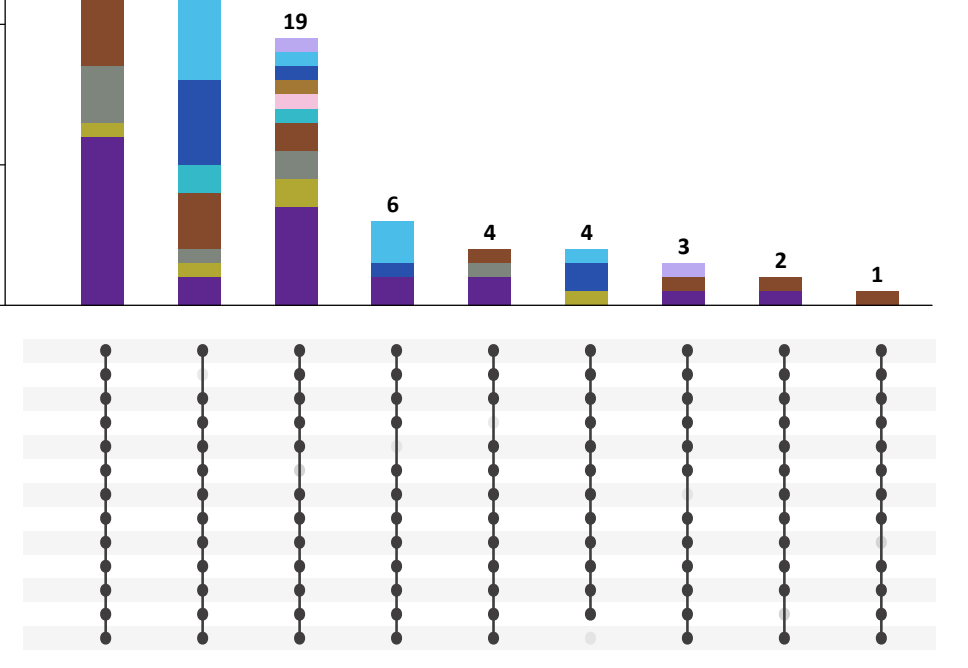


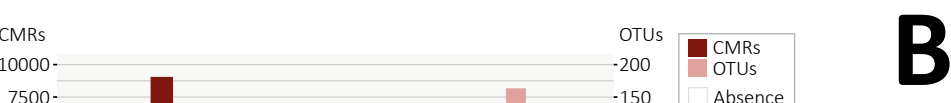

BK16S_846m

III r. IIII:

BK14S_129m

BK11S_10m

$\stackrel{\cong}{0}$ BK05S_1450m

BK22S_592m

BK04S_0.5m

BK03S_1081m

BK25s_323m

BK01S1_90m

BK01S2_15m

BK26S_1412m

BK30S_1381m
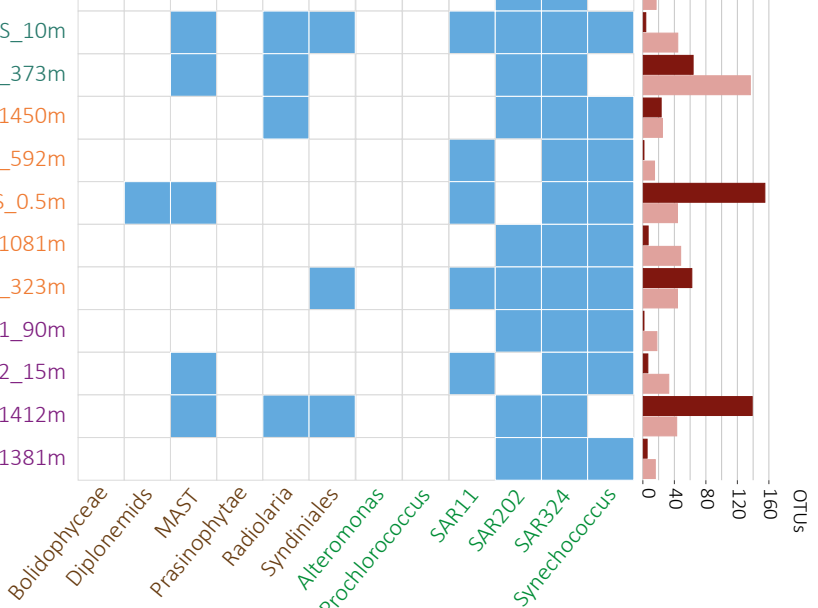

Eukaryotes

Prokaryotes

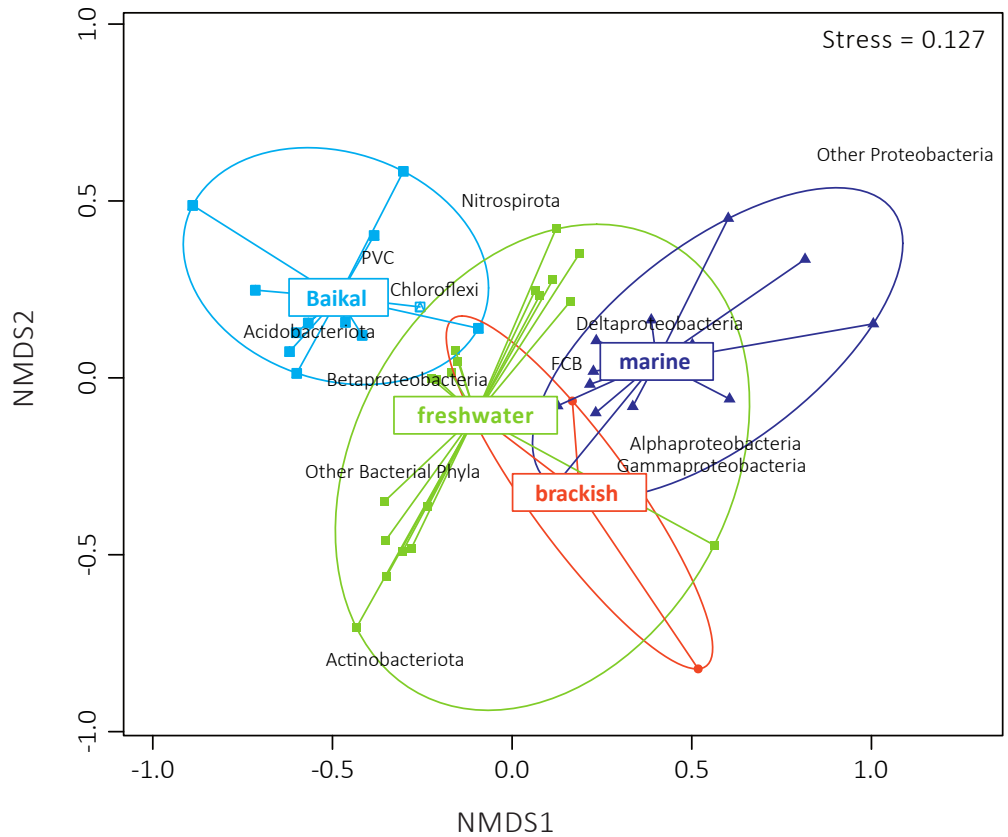

Lake Baikal

Siberia, Russia

North Yellow Sea, China

Caspian Sea

North Yellow Sea, China I

Changjiang Estuary

East China Sea*

Bering Sea

Changjiang Estuary

East China Sea

Lake Taihu Jiangsu, China

Bering Sea

Caspian Sea

Byers Peninsula +

Livingston Island

Maritime Antarctica

Kongsfiorden, Norway

Bering Sea

Caspian Sea I
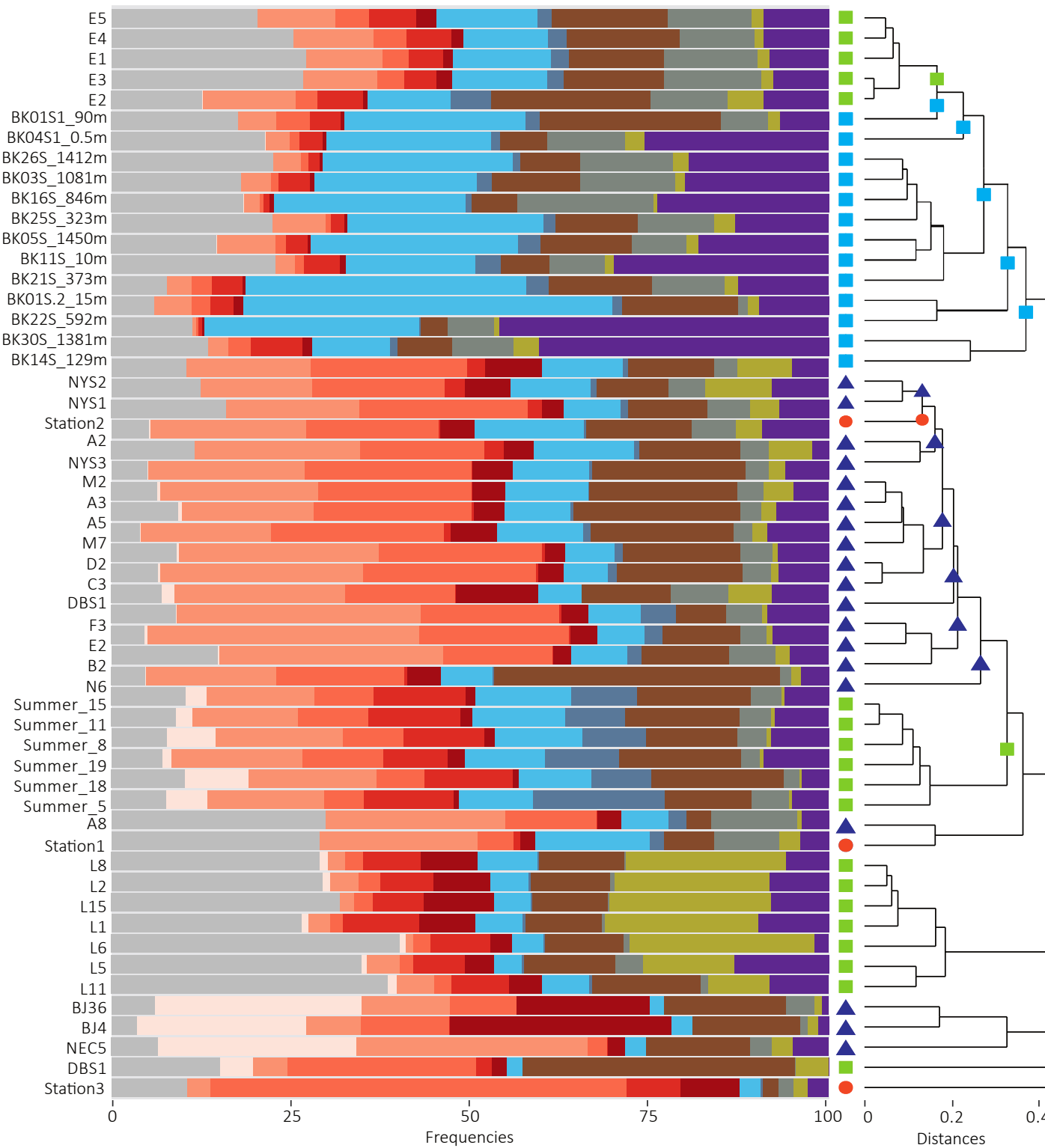

스
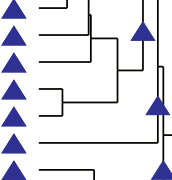

A

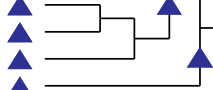

-

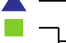

7

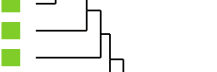

$\square$

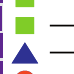

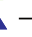

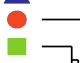

$\square$

$-$
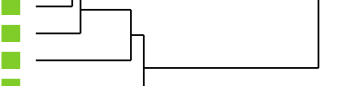

$\longrightarrow$
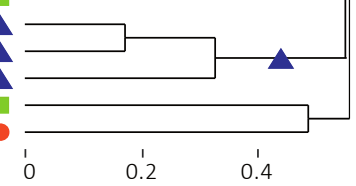

Other bacterial phyla

Other Proteobacteria

Deltaproteobacteria

Gammaproteobacteria
Betaproteobacteria

Alphaproteobacteria
PVC

Nitrospirota
FCB

Distances

Figure 2. Reboul et al 